\title{
Efecto de agentes remineralizantes a base de fosfato de calcio sobre la dentina
}

\author{
Effect of Calcium Phosphate-Based Remineralizing Agents on Dentin
}

Wilfredo Gustavo Escalante Otárola ${ }^{1 b}$, Gabriela Mariana Castro Núñez ${ }^{2 b, c}$, Milton Carlos Kuga ${ }^{3 b, c}$

D https://orcid.org/0000-0003-4879-3938

(iD https://orcid.org/0000-0002-2586-541X

(iD) https://orcid.org/0000-0001-5728-8293

\section{Resumen}

Objetivos: Evaluar los efectos de agentes remineralizantes a base de fosfato de calcio en la obliteración de túbulos dentinarios y dispersión de energía en la dentina cervical.

Material y métodos: Treinta especímenes de dentina cervical bovina fueron previamente erosionados (ácido clorhídrico al 0,6 \%, pH 2,3; $5 \mathrm{~min}$ ) y divididos aleatoriamente en 3 grupos $(\mathrm{n}=$ 10): Control - sin tratamiento, NP - Desensibilize Nano P (FGM ${ }^{\circledR}$ ) y RD - MI Paste Plus (Recaldent ${ }^{\mathrm{TM}}$ ). Los tratamientos fueron aplicados en cuatro sesiones con intervalos de 7 días. Los especímenes fueron analizados por microscopia electrónica de barrido (MEB) y espectroscopia de rayos $X$ de energía dispersiva (EDX). Resultados: Los grupos NP e RD mostraron mayor obliteración de túbulos dentinarios que el grupo control $(p<0,05)$. Las concentraciones de $\mathrm{Ca} / \mathrm{P}$ fueron similares entre todos los grupos evaluados. Conclusiones: Los agentes remineralizantes a base de fosfato de calcio demostraron ser una alternativa promisoria en la obliteración de túbulos dentinarios.

Palabras clave: dentina, erosión dental, hipersensibilidad dentinaria, microscopía electrónica

\section{Abstract}

Objectives: To assess the effects of phosphate-based remineralizing agents on obliteration of the dentinal tubules and energy dispersion in the cervical dentin.

Material and Methods: Thirty specimens of bovine cervical dentin were previously eroded $(0.6 \%$ hydrochloric acid, $\mathrm{pH} 2.3 ; 5 \mathrm{~min})$ and randomly divided into 3 groups $(\mathrm{n}=10)$ : Control - no treatment, NP - Desensitize Nano P (FGM®) and RD - MI Paste Plus (RecaldentTM). The treatments were applied in four sessions with intervals of 7 days. The specimens were analyzed by scanning electron microscopy (SEM) and energy dispersive X-ray spectroscopy (EDX). Results: The NP and RD groups showed major obliteration of the dentin tubules than the control group $(p<0.05)$. Ca / P concentrations were similar between all groups evaluated. Conclusions: Remineralizing agents based on calcium phosphate proved to be a promising alternative in obliteration of dentin tubules.

Keywords: dentin, dental erosion, dentin hypersensitivity, electron microscopy

${ }^{1}$ Investigador independiente

${ }^{2}$ Departamento Académico de Estomatología Rehabilitadora, Universidad Nacional Mayor de San Marcos, Lima, Perú ${ }^{3}$ Departamento de Odontología Restauradora, Universidad Estadual Paulista, São Paulo, Brasil

${ }^{a}$ Doctor Especialista en Ortodoncia y Ortopedia Maxilar

bDoctor en Odontología

'Docente 


\section{Introducción}

La hipersensibilidad dentinaria (HD) es descrita clínicamente como un dolor agudo causado por la excitación de las fibras nerviosas A delta conectadas a la dentina expuesta y sus túbulos. Esto debido a la estimulación química, térmica, osmótica, evaporativa o táctil. ${ }^{1}$ La teoría hidrodinámica de Brannstrom et al. ${ }^{2}$ proporciona la explicación más presumible para el mecanismo de la HD. En la actualidad, existe una tendencia en dos enfoques basados en esa teoría. El primero de ellos, obliterando túbulos dentinarios abiertos; y el segundo, bloqueando la actividad nerviosa de las fibras A delta. ${ }^{1}$ Los protocolos desensibilizantes actuales son direccionados al tratamiento de la HD por medio de la formación de precipitados minerales insolubles dentro de los túbulos dentinarios utilizando agentes remineralizantes. $^{3}$

El agente desensibilizante que contiene fosfato de calcio nanoparticulado en forma de hidroxiapatita ha sido recomendado tanto para remineralizar los tejidos dentarios como para el tratamiento de la HD, ya que forman una película de protección sobre la dentina. ${ }^{4,5}$ El mecanismo de acción de este hecho está posiblemente relacionado a la capacidad de estabilizar los iones de calcio y fosfato en un estado amorfo en la estructura dental; similarmente, esto ocurre con el CPPACP. $^{6-8}$ La aplicación del CPP-ACP está indicada para la remineralización de dientes, además de sus propiedades antierosivas. ${ }^{9-11}$

Por tal motivo, el objetivo de este estudio fue evaluar el efecto de los protocolos remineralizantes dentarios que contienen nanopartículas de fosfato de cálcio (Desensibilize NanoP) o fosfopéptido de caseína/fosfato de calcio amorfo (MI Paste Plus Recaldent ${ }^{\mathrm{TM}}$ CPP-ACP) sobre la dentina cervical por medio de la obliteración de túbulos dentinários y análisis de dispersión de energía en comparación con el grupo de control. La hipótesis nula fue que los protocolos remineralizantes no tienen efecto sobre la dentina según los parámetros evaluados.

\section{Material y métodos}

Este estudio fue aprobado por el Comité de Ética en animales de la Facultad de Odontología de Araraquara, Universidad Estatal de Paulista ( $\left.N^{\circ} 30 / 2018\right)$.

\section{Preparación de especímenes}

Treinta incisivos bovinos con anatomía coronaria y radicular similares fueron almacenados en solución de Thymol al $0,1 \%$ a $4{ }^{\circ} \mathrm{C}$ hasta su uso. Fueron obtenidos treinta especímenes $(10 \mathrm{~mm}$ de largo $\times 10 \mathrm{~mm}$ de ancho $\times 5 \mathrm{~mm}$ de altura) de la porción cervical (coronal y radicular) de los dientes, usando una máquina de corte de tejidos duros (Isomet 100, Buehler, Lake Bluff, IL) bajo constante refrigeración. La superficie vestibular de los fragmentos fue pulida en una máquina de pulido (DP-10; Panambra, Struers, Ballerup, DI) usando la secuencia de lijas de granulación \#600 y \#1200 por 20 segundos cada una para exponer la dentina cervical.

La superficie dentinaria fue previamente erosionada con $\mathrm{HCl}$ a $0,6 \%$ y $\mathrm{pH}$ de 2,3 por inmersión de 5 minutos. Luego, los especímenes fueron lavados con agua destilada y almacenados en saliva artificial $\left(0,375 \mathrm{~g} / 1 \mathrm{CaCl}_{2} \cdot 2 \mathrm{H}_{2} \mathrm{O} ; 0,125 \mathrm{~g} / \mathrm{l} \mathrm{MgCl} \cdot 6 \mathrm{H}_{2} \mathrm{O}\right.$; $1,2 \mathrm{~g} / \mathrm{l} \mathrm{KCl} ; 0,85 \mathrm{~g} / \mathrm{l} \mathrm{NaCl} ; 2,5 \mathrm{~g} / \mathrm{l} \mathrm{NaHPO}_{4}$. $12 \mathrm{H}_{2} \mathrm{O} ; 1 \mathrm{~g} / \mathrm{l}$ ácido sórbico; $5 \mathrm{~g} / \mathrm{l} \mathrm{de}$ hidroxietilcelulosa de sodio y $43 \mathrm{~g} / \mathrm{l}$ de solución de sorbitol a $37^{\circ} \mathrm{C}$ hasta su uso.

\section{Tratamiento}

En la Tabla 1 se muestra la composición química de los agentes remineralizantes utilizados en el estudio. 


\section{Tabla 1}

Composición de los materiales usados en este estudio

\begin{tabular}{lll}
\hline Grupo & Composición & Fabricante \\
\hline $\mathrm{CO}-$ Control & Agua destilada & - \\
$\mathrm{NP}-$ Desensibilize & Fosfato de calcio (nano-HAP), nitrato de & FGM, Joinville, Brasil \\
Nano P & potasio y 9000 ppm fluoruro de sodio. & \\
$\mathrm{RD}-\mathrm{MI}$ Paste Plus & CPP-ACPF (Caseína Fosfopeptido-Fosfato GC, Tokyo, Japón \\
Recaldent ${ }^{\mathrm{TM}}$ & de Calcio Amorfo Fluoruro), Glicerina, D- \\
& Sorbitol, CMC-Na, Propileno glicol, Dióxido de \\
& silicona, Dióxido de titanio, Xilitol, Ácido \\
& fosfórico, Saborizantes, Sacarina sódica, \\
& Ethyl p-hydroxybenzoate, Propyl p- \\
& hydroxybenzoate, Butyl p-hydroxybenzoate. \\
& 900 ppm Fluoruro. \\
\hline
\end{tabular}

Diez especímenes fueron usados para cada protocolo $(n=10)$, los cuales fueron aplicados según recomendaciones de los fabricantes para los análisis con Microscopía Electrónica de Barrido (MEB) y Espectroscopía de Rayos X de Energía Dispersiva (EDX). Los protocolos evaluados fueron los siguientes:

- Grupo control: Los especímenes se mantuvieron en agua destilada durante 10 minutos.

- Grupo NP: El producto que contiene nanopartículas de fosfato de calcio (Desensibilize Nano P; FGM, Joinville, SC, Brasil) fue aplicado con un microbrush (KG Sorensen, São Paulo, SP, Brasil) y friccionado sobre la superficie dentinaria con una taza de goma (KG Sorensen, São Paulo, SP, Brasil) con micromotor de baja velocidad (500 rpm por 10 segundos). Luego, el material se dejó en contacto con la dentina durante 5 minutos y se enjuagó con $10 \mathrm{~mL}$ de agua destilada, de acuerdo con las recomendaciones del fabricante.

- Grupo RD: El material que contiene CPP-ACPF (MI Paste Plus Recaldent ${ }^{\mathrm{TM}}$; GC Company; Japón) fue aplicado con microbrush (KG Sorensen, São Paulo, SP, Brasil) y dejado en contacto con la superficie dentinaria durante 3 minutos. Los residuos fueron enjuagados con $10 \mathrm{~mL}$ de agua destilada.
Los especímenes fueron sometidos a 4 sesiones de tratamiento remineralizante, con un intervalo de 7 días. Durante los 28 días de experimento, los especímenes fueron mantenidos en saliva artificial a $37^{\circ} \mathrm{C}$.

\section{Análisis con Microscopía Electrónica de Barrido (MEB)}

Tras completar los tratamientos descritos, los especímenes fueron almacenados en saliva artificial por 24 horas. Luego, estos fueron deshidratados en una cámara cerrada con sílice coloidal por 7 días. Los especímenes fueron posicionados en stubs metálicos, para ser metalizados con oro coloidal (ciclo simple - 120 segundos) y examinados en MEB con JEOL 6060 (JEOL 6060; JEOL Ltda, Tokyo, Japón) operado a 20 $\mathrm{kV}^{7,12}$

Se obtuvieron imágenes representativas de la región central de cada espécimen, con un aumento de 2000x para contar los túbulos dentinarios abiertos. Todas las imágenes fueron obtenidas por un solo operador.

Dos examinadores debidamente calibrados, quienes utilizaron la prueba de Kappa ponderada $(\mathrm{k}=0,82)$, realizaron el conteo de túbulos dentinarios abiertos independientemente. 


\section{Análisis con espectroscopía de rayos $X$ de} energía dispersiva (EDX)

Cuatro especímenes de cada protocolo se cubrieron con carbón (BalTec SCD 004 Sputter Coater; Balzers, Vaduz, Liechtenstein) a $15 \mathrm{kV}$ durante 180 segundos. Luego, estos especímenes fueron analizados por EDX (JEOL 6060; JEOL Co., Tokio, Japón) para evaluar los principales componentes químicos presentes en la superficie de la dentina después de realizar los protocolos del experimento.

\section{Análisis estadístico}

Inicialmente, los datos obtenidos fueron sometidos a la prueba de Shapiro-Wilk para determinar la normalidad de los datos. El conteo de los túbulos dentinarios abiertos (MEB) se sometió a las pruebas de KruskalWallis y Dunn con un nivel de significancia de $\alpha=5 \%$.

\section{Resultados}

\section{Análisis con MEB}

En la Tabla 2 se muestran los resultados del conteo de túbulos dentinarios abiertos de la superficie de dentina erosionada después de los tratamientos remineralizantes. Los grupos NP y RD presentaron la menor incidencia de túbulos dentinarios abiertos en comparación con el grupo CO $(p<0,05)$.

\section{Tabla 2}

Número de túbulos dentinarios abiertos

\begin{tabular}{lllll}
\hline & & CO & NP & RD \\
\hline \multirow{2}{*}{$\begin{array}{l}\text { Túbulos dentinarios } \\
\text { abiertos }\end{array}$} & Promedio & $38,14^{\mathrm{b}}$ & $20,43^{\mathrm{a}}$ & $21,71^{\mathrm{a}}$ \\
& DS & 9,35 & 8,04 & 4,39 \\
\hline
\end{tabular}

Nota. Letras diferentes en la misma linea indican diferencias significativas $(P<0,05)$. DS: desviación estándar; CO: Control; NP: Desensibilize Nano P; RD: MI Paste Plus Recaldent.

La Figura 1 muestra una imagen representativa de la obliteración de los túbulos dentinarios (2000x) para cada grupo evaluado.

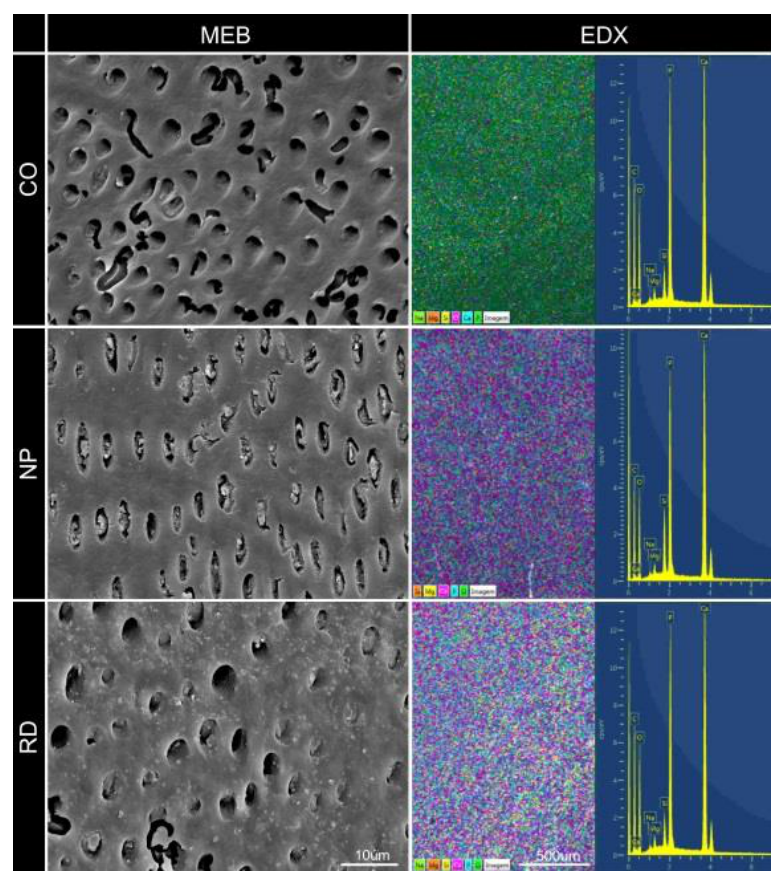

Figura 1. Imagen representativa de superficie dentinaria a 2000x (MEB) y del análisis con espectroscopía de rayos $X$ de energía dispersiva (EDX), después de los tratamientos evaluados. CO: Control; NP: Desensibilize Nano P; RD: MI Paste Plus Recaldent. Escalas $10 \mu \mathrm{m}$ y 500 um. 


\section{Análisis con EDX}

Los grupos NP, RD y CO mostraron los siguientes valores de concentración entre la relación atómica $\mathrm{Ca} / \mathrm{P}$ : $(\mathrm{CO}), \mathrm{Ca} / \mathrm{P}=1,80 ;(\mathrm{NP}), \mathrm{Ca} / \mathrm{P}=1,88 ;(\mathrm{RD}) \mathrm{Ca} / \mathrm{P}=1,80$. Además, mostraron la presencia de silicio $(\mathrm{Si})$, magnesio $(\mathrm{Mg})$ y sodio $(\mathrm{Na})$.

La Figura 1 muestra una imagen representativa de la composición precipitada. Por otro lado, la Figura 2 muestra la relación entre $\mathrm{Ca} / \mathrm{P}$ después del uso de los materiales evaluados.

Figura 2. Imágenes del análisis con espectroscopía de rayos $X$ de energía dispersiva (EDX). Ca: calcio; P: fosforo; Ca/ P relación atómica. CO: Control; NP: Desensibilize NanoP; RD: MI Paste Plus Recaldent.

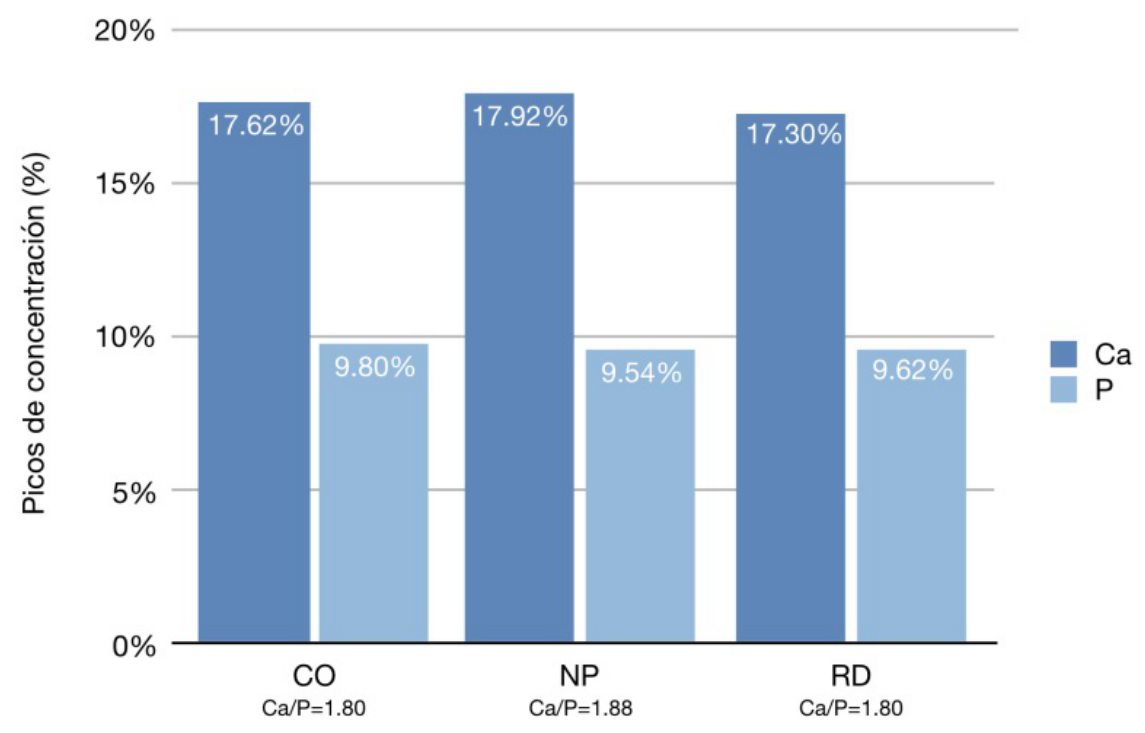

\section{Discusión}

Como los tratamientos más aceptados para la temible hipersensibilidad dentinaria tienen como objetivos la obstrucción de los túbulos dentinarios $y$, si es posible, la protección de la dentina intertubular asociada con el bloqueo de la neurotransmisión por los nociceptores de las fibras delta tipo A ubicadas en la interfaz de la dentina y la pulpa dental; los agentes remineralizantes representan una interesante estrategia de tratamiento. ${ }^{2,3}$ En el presente estudio, ambos protocolos (NP y RD) demostraron una menor incidencia de túbulos dentinarios abiertos que la proporcionada por CO. Por lo tanto, la hipótesis nula fue rechazada.

En el análisis obtenido en EDX, se observó que el precipitado formado sobre la dentina erosionada previamente aumentó en su concentración de calcio y fosforo después del tratamiento con los agentes remineralizantes evaluados. Este hecho puede deberse a la relación directa existente entre la estabilidad química de la película formada con su disolución y/o solubilización. ${ }^{3,13,4}$

El RD contiene en su composición química el fosfopéptido CCP-ACP (fosfato cálcico-fosfato de caseína-amorfo) y 900 ppm de flúor. ${ }^{15,16}$ Cuando se usa en el sustrato dental, el CPP-ACP proporciona la formación de una alta concentración de fluorapatita, el cual es un mineral más estable e insoluble que la hidroxiapatita. ${ }^{4,78}$ EI NP también tiene un mecanismo de acción similar al $R^{6,19}$, pero más relacionado con la deposición de $\mathrm{Ca}$ y $\mathrm{P}$ precipitados, con características amorfas, que la fluorapatita misma en cuanto a la estructura dental se refiere. ${ }^{4,6,20}$ Sin embargo, NP también tiene nitrato de potasio en su formulación química. ${ }^{20,21}$

Aunque el compuesto con silicato de calcio y fosfato de sodio utilizado en el grupo NR indujo la formación de precipitados en la dentina, también demostró un elevado número de túbulos dentinarios abiertos. Esto 
se puede explicar por la estrategia de tratamiento empleada en este estudio, ya que el fabricante recomienda el uso domiciliario y diario del material. ${ }^{22,23}$

El grupo KF tratado con nitrato de potasio al $5 \%$ asociado con $\mathrm{NaF}$ al $2 \%$ mostró los peores resultados antierosivos y fue similar al grupo de control. EI nitrato de potasio actúa específicamente sobre la polaridad de la fibra nerviosa estimulada durante la $\mathrm{DH}$, ${ }^{24,25}$ y no hay evidencia de que pueda formar precipitados mineralizados en la superficie dentaria.

Dado que el RD y el NP son agentes remineralizantes con mecanismo y acción similares; ambos agentes, proporcionan una formación satisfactoria de precipitados en la dentina intertubular. Asimismo, potencial para obstruir las entradas del túya que estos agentes presentan un buen bulo de dentina, pueden ser una alternativa interesante para ayudar en el tratamiento de la hipersensibilidad dentinaria.

\section{Conclusión}

Los agentes remineralizantes que contienen fosfato de calcio (CPP-ACPF y n-HAP) son alternativas eficientes para la obliteración de los túbulos dentinarios.

\section{Declaración de intereses}

Los autores declaran que no tienen conflicto de intereses.

\section{Referencias}

1. Gillam DG, Mordan NJ, Newman HN.The Dentin Disc surface: a plausible model for dentin physiology and dentin sensitivity evaluation. Adv. Dent. Res. 1 nov 1997; 11(4): 487-501. Doi: $10.1177 / 08959374970110041701$

2. Brannstrom M. The hydrodynamic theory of dentinal pain: sensation in preparations, caries, and the dentinal crack syndrome. J Endod. Oct 1986; 12(10) 453-7. Doi: 10.1016/S00992399(86)80198-4

3. Berkathullah M, Farook MS, Mahmoud $O$. The Effectiveness of Remineralizing A g e $n$ t s o $n$ D e $n t i n$ a Permeability.BioMed Res.12 sep 2018;

\section{8:12. Doi: 10.1155/2018/4072815}

4. Carvalho FG, Brasil VL, Silva Filho TJ, Carlo HL, Santos RL, Lima BA, Protective effect of calcium nanophosphate and CPPACP agents on enamel erosion. Brazilian Oral Res. Nov-Dic 2013; 27(6): 463-70. D O I : $10.1590 / S 1806$ 83242013000600004

5. Mantzourani M, Sharma D. Dentine sensitivity: past, present and future. J Dent. Jul 2013; 41 Suppl 4: S3-17. doi: 10.1016/S0300-5712(13)70002-2. PMID: 23929643.

6. Comar LP, Souza BM, Gracindo LF, Buzalaf MA, Magalhaes AC. Impact of experimental nano-HAP pastes on bovine enamel and dentin submitted to a $\mathrm{pH}$ cycling model. Braz Dent J. 6 may 2013; 24 ( 3 ): $273-8$. Doi:10.1590/01036440201302175

7. Kuga MC, So MV, De Faria-junior NB, Keine KC, Faria G, Fabricio S, Matsumoto MA. Persistence of resinous cement residues in dentin treated with different chemical removal protocols. Microsc Res Tech. Jul 2012; 75(7): 982-5. Doi: 10.1002/jemt.22023

8. Van Meerbeek B, Yoshihara K, Yoshida Y, Mine A, De Munck J, Van Landuyt KL. State of the art of self-etch adhesives, Dent Mater. Ene 2011; 27(1): 17-28. Doi: 10.1016/j.dental.2010.10.023.

9. Pamir T, Dalgar H, Onal B. Clinical evaluation of three desensitizing agents in relieving dentin hypersensitivity. Oper Dent. 1 nov 2007; 32(6): 544-8. Doi: 10.2341/07-5.

10. Schmidlin PR, Sahrmann P. Current management of dentin hypersensitivity. Clin Oral Investig [Internet]. Mar 2013.17 Suppl 1: S55-9. Doi: 10.1007/s00784012-0912-0

11. Walters PA. Dentinal hypersensitivity: a review. Contemp Dent Pract.15 may 2005; 6(2):107-17. PMID: 15915210

12. Escalante-Otarola WG, Castro-Nunez GM, Jordao-Basso KCF, Guimaraes BM, Palma-Dibb RG, Kuga MC. Evaluation of dentin desensitization protocols on the dentinal surface and their effects on the dentin bond interface. J. Dent. Ag 2018; $75: 98-104$. D O i : 10.1016/j.jdent.2018.06.002. 
13. Cochrane NJ, Reynolds EC. Calcium phosphopeptides -- mechanisms of action and evidence for clinical efficacy. Adv Dent Res. Sept 2012; 24(2):41-7. Doi: $10.1177 / 0022034512454294$.

14. Sun Y, Li X, Deng Y, Sun JN, Tao D, Chen $H$, Hu Q, Liu R, Liu W, Feng X, Wang J, Carvell $M$, Joiner $A$. Mode of action studies on the formation of enamel minerals from a novel toothpaste containing calcium silicate and sodium phosphate salts. J Dent. Jun 2014; 42 Suppl 1: S30-8. Doi: 10.1016/S03005712(14)50005-X.

15. Mehta D, Gowda V, Finger WJ, Sasaki K, Randomized placebo-controlled study of the efficacy of a calcium phosphate containing paste on dentin hypersensitivity. Dent Mat. Nov 2015; 31(11): $1298-303$. D o i : 10.1016/j.dental.2015.08.162.

16. Komabayashi T, Imai Y, Ahn C, Chow LC, Takagi S. Dentin permeability reduction by a sequential application of calcium and fluoride-phosphate solutions. J Dent. Sept 2010; 38(9): 736-41. Doi: 10.1016/j.jdent.2010.05.019.

17. Tenuta LM, Cury JA. Fluoride: its role in dentistry. Braz Oral Res. 2010; 24 Suppl 1: 9-17. Doi: 10.1590/s180683242010000500003.

18. Alencar CM, Leite KLF, Ortiz MIG, Magno MB, Rocha GM, Silva CM \& Maia LC (2020) Morphological and Chemical Effects of In-Office and At-Home Desensitising Agents Containing Sodium Fluoride on Eroded Root Dentin. Archives of Oral Biology. Feb 2020; 10:104619. D 10.1016/j.archoralbio.2019.104619.

19. Souza BM, Comar LP, Vertuan M, Fernandes Neto C, Buzalaf MA, Magalhaes AC. Effect of an Experimental Paste with Hydroxyapatite Nanoparticles and Fluoride on Dental Demineralisation and Remineralisation in situ. Caries research. Agto 2015; 49(5):499-507. Doi: $10.1159 / 000438466$.
20. Santos LF, Torres CR, Caneppele TM, Magalhaes AC, Borges AB. Effect of home-bleaching gels modified by calcium and/or fluoride and the application of nano-hydroxyapatite paste on in vitro enamel erosion susceptibility. Acta Odontol Scand. 2016;74(2):121-6. Doi: 10.3109/00016357.2015.1053150.

21. Medeiros IC, Brasil VL, Carlo HL, Santos $\mathrm{RL}$, De Lima BA, De Carvalho FG. In vitro effect of calcium nanophosphate and high-concentrated fluoride agents on enamel erosion: an AFM study. International Journal of Paediatric Dentistry. May 2014; 24(3):168-174. Doi: 10.1111/ipd.12046.

22. Hornby K, Ricketts SR, Philpotts CJ, Joiner A, Schemehorn B \& Willson R. Enhanced enamel benefits from a novel toothpaste and dual phase gel containing calcium silicate and sodium phosphate salts. J Dent. Jun 2014; 42 Suppl 1:S3945. Doi: 10.1016/S0300-5712(14)500061.

23. Sun $Y$, Li X, Deng $Y$, Sun JN, Tao D, Chen $\mathrm{H}, \mathrm{Hu} \mathrm{Q}$, Liu R. Liu W, Feng X, Wang J. Carvell $M$ \& Joiner $A$. Mode of action studies on the formation of enamel minerals from a novel toothpaste containing calcium silicate and sodium phosphate salts. J Dent. Jun 2014; 42 Suppl 1:S30-8. Doi: 10.1016/S03005712(14)50005-X.

24. Wang $Y$, Gao J, Jiang T, Liang S, Zhou Y \& Matis BA. Evaluation of the Efficacy of Potassium Nitrate and Sodium Fluoride as Desensitizing Agents During Tooth Bleaching treatment-A Systematic Review and Meta-Analysis. J Dent. Agst $2015 ; 43$ ( 8$): 913-23$. D o i : 10.1016/j.jdent.2015.03.015.

25. João-Souza SH, Scaramucci T, Bühler Borges A, Lussi A, Saads Carvalho T \& Corrêa Aranha AC. Influence of desensitizing and anti-erosive toothpastes on dentine permeability: An in vitro study. J Dent. Oct 2019; $89: 103176$. d o i : 10.1016/j.jdent.2019.07.014.
Correspondencia:

wilfredoescalante@gmail.com
Fecha de recepción : 30 de julio de 2020

Fecha de aceptación : 15 de septiembre de 2020 